\title{
The association of gonorrhoea and syphilis with premature birth and low birthweight
}

\author{
G G G Donders, J Desmyter, D H De Wet, F A Van Assche
}

From the Department of Obstetrics and Gynaecology Katholieke Universiteit Leuven, Belgium G G G Donders F A Van Assche

Department of Microbiology, Katholieke Universiteit Leuven, Belgium

J Desmyter

Department of Obstetrics and Gynaecology, University of Pretoria, South-Africa G G G Donders G H De Wet

Correspondence to: G Donders, Department of Obstetrics \& Gynaecology, Gasthuisberg University Hospital, Katholieke Universiteit Leuven Herestraat 49 3000 Leuven, Belgium Accepted for publication 26 October 1992

\begin{abstract}
Objective-Provide evidence from prospective data that Neisseria gonorrhoeae may be an important cause of premature delivery and low birth weight in areas with high prevalence of genital infections.
\end{abstract}

Setting-Department of Obstetrics and Gynaecology, Kalafong University Hospital, Pretoria, South Africa in collaboration with the Departments of Microbiology and of Gynaecology and Obstetrics, Katholieke Universiteit, Leuven, Belgium.

Subjects-Two hundred and fifty six consecutive black pregnant women were examined during the first antenatal visit, and one to four weeks later a second culture for $\boldsymbol{N}$ gonorrhoeae was taken at random in 67 of them. Hundred and sixty seven were analysable, 75 were lost to follow up.

Methods-After obtaining detailed clinical history, an endocervical specimen for $\boldsymbol{N}$ gonorrhoeae culture (Thayer-Martin) and $C$ trachomatis antigen detection (Chlamydiazyme (R)) was taken. Syphilis was diagnosed when both reactive plasma protein (RPR) and $T$ pallidum haemagglutination inhibition assay (TPHA) were positive. Prematurity was defined as delivery at less than 37 gestational weeks. Results-Infection with $N$ gonorrhoeae $(n=9)$ and untreated syphilis $(n=7)$ were both associated with prematurity and low birth weight. After multi-variate regression analysis, age-adjusted parity, late sexual debut, number of recent sexual partners, infection with $N$ gonorrhoeae and infection with syphilis revealed significant associations with low birth weight. However, infection with $C$ trachomatis, presence of abundant vaginal discharge, social class, Trichomonas vaginalis infection, gestational weeks at first antenatal visit and number of previous miscarriages did not reveal such an association. Attributable risk of untreated gonorrhoea for premature birth was $72 \%$ and routine cultures were cost-benefit efficient.

Conclusions-At least in countries where the prevalence is high, genital infections as well as the risk factors for acquiring them (young age, late sexual debut, number of recent partners) play a major role in the aetiogenesis of prematurity and low birth weight. $N$ gonorrhoeae is a main contributor, and in high prevalence areas it should be routinely looked for and treated for during pregnancy.

\section{(Genitourin Med 1993;69:98-101)}

Introduction

Identifying preventable causes of premature birth and premature rupture of membranes (PROM) remains a major challenge in the reduction of perinatal morbidity and mortality. Prematurity, among other complications, accompanies at least $20 \%$ of pregnancies during which syphilis was diagnosed. Maternal infections with Chlamydia trachomatis, Group B streptococcus, Ureaplasma urealyticum, Mycoplasma hominis, Trichomonas vaginalis, Staphylococcus aureus and bacterial vaginosis have statistical associations with adverse pregnancy outcome. ${ }^{1-6}$

Case reports ${ }^{78}$ and case-control studies ${ }^{9-11}$ also suggest an association of maternal gonorrhoea with premature delivery and chorioamnionitis, but prospective data are extremely scarce. ${ }^{12}$

At least one prenatal cervical culture for Neisseria gonorrhoeae is recommended during pregnancy. ${ }^{13}$ In African countries, in spite of prevalence rates of gonorrhoea as high as $5 \%$ to $20 \%,{ }^{14} 15$ such preventive measures are largely non-existent ${ }^{16}$ and when a diagnosis is made, it may be difficult to reach the patient for treatment.

In this study in an African setting, where the diagnosis of syphilis and gonorrhoea was made at the first antenatal visit, we have prospectively related both infections with prematurity and low birth weight.

\section{Material and methods}

In 1988 we examined 256 consecutive black pregnant women at Kalafong Hospital, Pretoria, South Africa at their first antenatal visit for cervical $N$ gonorrhoeae and for syphilis serology. This was part of a larger study of cervicitis in pregnancy. ${ }^{17}$

For culture of gonococci, the cotton-tipped swab was transferred after three rotations in the endocervical canal to Stuart's transport medium according to Amies, plated the same day on Thayer-Martin agar, and colonies were examined after two days at $37^{\circ} \mathrm{C}$. Duplicate swabs were taken at random from 67 women during the next visit, from one week to four weeks later.

Sera were examined by the Rapid Plasma 
Reagent flocculation test (RPR, Welcome) and by the Treponema pallidum haemagglutination inhibition test (TPHA, Cambridge Biomedical). Syphilis was only diagnosed when both assays were positive.

Of the 256 pregnancies 167 delivered (birthweight $>500 \mathrm{~g}$ ) at Kalafong Hospital and were included in the analysis. Seventyfive women who did not return to Kalafong Hospital and about whom we have no information, nine pregnancies ending in a spontaneous abortion, one with a uterus didelphus and four with twins were excluded from analysis of pregnancy outcome. No differences were noted between the studied group and the group of excluded women, apart from a slightly lower infection rate of $N$ gonorrhoeae and $C$ trachomatis, most likely due to lack of duplicate swabs in the lost-to-followup group (table 1).

Syphilis screening had been routinely performed before this study, but it remained difficult to track positive pregnant women for treatment. Similarly it was not possible to enforce treatment of gonorrhoea during this study. Thus only eight of the 15 analysable women with positive syphilis serology, and none of the nine women with positive cultures for $N$ gonorrhoeae, were reached for treatment during pregnancy.

None of the 256 women had antibodies against HIV-1 and HIV-2 (Abbott recombinant combined HIV-1/HIV-2 enzyme

Table 1 Comparison of study population $(n=167)$ versus group of patients excluded from analysis (see text) $(n=89)$.

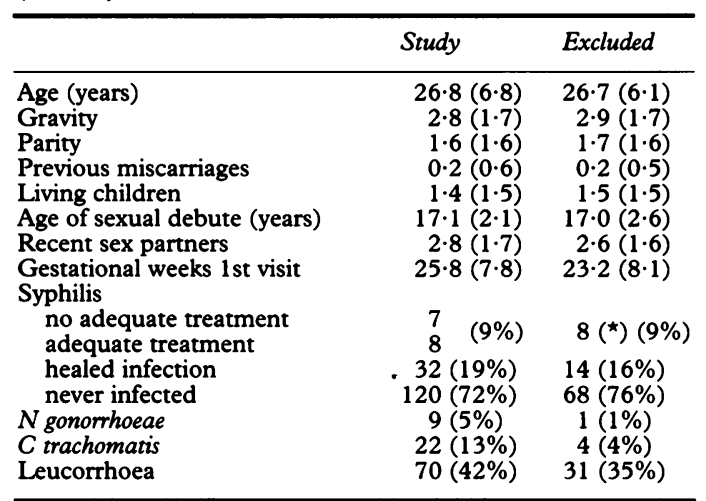

In brackets standard deviation (SD) is given for mean values or percentage (\%) for numbers.

*As no information on treatment was available in the lost-tofollow-up group, percentage is referring to total infected by $\mathrm{T}$ pallidum.

Table 2 Stepwise multi-variate regression analysis of 10 variables influencing birthweight. $R$-squared of regression model including the first five variables: 0.193

\begin{tabular}{llll} 
& $\begin{array}{l}\text { Standardised } \\
\text { regression } \\
\text { coefficient }\end{array}$ & $\begin{array}{l}\text { Additive } \\
R^{2}\end{array}$ & Probability \\
\hline$N$ gonorrhoeae & -0.16 & 0.022 & 0.039 \\
Syphilis & -0.15 & 0.020 & 0.049 \\
Parity category (*) & 0.26 & 0.058 & 0.001 \\
Age of sexual debute (years) & -0.23 & 0.049 & 0.002 \\
Sexual partners & -0.17 & 0.028 & 0.019 \\
Leucorrhoea & & 0.016 & 0.077 \\
Social class & & 0.013 & 0.1 \\
Weeks at first visit & 0.012 & 0.1 \\
Miscarriages & 0.003 & 0.4 \\
C trachomatis & & 0.001 & 0.7 \\
\hline
\end{tabular}

*Parity category: maternal age $<25$ years and parity $<3$ or maternal age $>=25$ and parity $<4$ is defined as low parity category. ${ }^{18}$ immuno-assy, or EIA). An EIA for the presence of Chlamydia trachomatis antigen on the endocervix was performed on stored samples according to the manufacturer's instructions (Chlamydiazyme Abbott).

Statistical analysis of the basic frequency tables was made by the chi square test and by the Fisher's exact test when the former was not appropriate. Relative risk with $95 \%$ confidence intervals and significance levels were given when relevant. Confounding was evaluated by estimating the Mantel-Haenszel relative risk. Independence of associations with birthweight was tested by multi-variate stepwise regression analysis. In order to account for the interaction between age and parity, two age-adjusted parity categories were used (table 2). ${ }^{18}$ Means of continuous variables with a standard normal distribution were compared by Student's $t$ test.

\section{Results}

Fifteen women had evidence of recent syphilis $(9 \%)$. Of these, eight women had been adequately treated, four were not treated at our institution and could not recall having had treatment elsewhere, and three recalled they were definitely not treated. In analysing the association of "untreated syphilis" with pregnancy outcome, the latter seven were taken as one group. Thirteen percent of the women had positive chlamydia antigen and nine $(5.4 \%)$ had untreated $N$ gonorrhoeae during pregnancy.

Seventeen percent delivered before completion of 37 gestational weeks. After multivariate regression analysis, younger women, women having multiple recent partners (during last six months) and women with late sexual debut had babies with lower birth weight (table 2). The interval between sexual debut and the index pregnancy was reciprocally linked to birthweight $(p=0 \cdot 04)$. Other sociodemographic or obstetric characteristics were not associated with low birth weight (table 2) or with higher prematurity rates (data not shown).

Women with $C$ trachomatis had more premature births ( $27 \%$ vs $16 \%)$, but this tendency was not significant (RR 2.0, C.I.95\% $0 \cdot 6-6 \cdot 1$ ) (table 3). The isolation of $N$ gonorrhoeae on the other hand, was strongly associated with a premature birth (RR 6.0, C.I.95\% 1.5-34.0) and with lower birth weight (2252 vs $2970 \mathrm{~g}, p<0.005)$, a tendency which remained unchanged after multi-variate analysis (table 2).

Of the 67 duplicate specimens three $N$ gonorrhoeae culture pairs were concordant positive, 60 were concordant negative and four $(6 \%)$ were discordant. Of the four discordant specimens the swab taken first was negative in three cases and the swab taken second was negative in one. The mean time lag between first and second culture specimen was only $2.5 \pm 1.5$ weeks, indicating that neither reinfection nor treatment was likely to have intervened. In the subgroup with known perinatal 
outcome the mean birthweight was $2313 \mathrm{~g}$ when both maternal duplicate specimens were positive $(n=3)$, compared to $2947 \mathrm{~g}$ when only one of the duplicate swabs was positive $(n=3, p 0 \cdot 2)$. One $(8 \%)$ of 13 gonococcal isolates showed in vitro resistance to penicillin.

In general, the finding of a positive $R P R$ and TPHA was not as such associated with an increased risk for premature delivery or low birth weight (table 3). However, among those who received no appropriate treatment, positive RPR was associated with both prematurity (RR 4.8, C.I.95\% 4.2-10.5) and low birth weight (2131 vs $2990 \mathrm{~g}, p=0.02)$. After multi-variate analysis this association remained significant (table 2 ).

Both untreated syphilis in the absence of gonorrhoea and untreated gonorrhoea in the absence of syphilis resulted in lower birthweight and increased prematurity (tables 2 and 4). When both gonorrhoea and syphilis occurred simultaneously, however, the risk for the newborn to have a low birthweight or to be born prematurely was higher than with either infection alone (table 4).

Among the women with gonorrhoea, the risk for premature birth could be reduced by $72 \%$ (C.I.95\% 0.62-0.87), should there be no gonorrhoea present (attributable risk among the exposed). Likewise $13 \%$ (C.I.95\% $0.05-0.20$ ) less prematurity could have resulted when gonorrhoea could be completely eradicated in this population (attributable risk among the population).

\section{Discussion}

The present data confirm that early detection and adequate treatment of syphilis during or before pregnancy remains a major challenge in high-prevalence countries. Our data add $N$ gonorrhoeae to the list of possible contributors to elevated prematurity rates and low birth weight. Like $C$ trachomatis, ${ }^{194} N$ gonorrhoeae may in certain circumstances lead to chorioamnionitis, prematurity and growth retardation, even with intact foetal membranes. ${ }^{79}$

Besides syphilis and $N$ gonorrhoeae infection, low age-adjusted parity, multiple recent sex partners and late sexual debut were risk factors for lower birth weight. While the former two were since long recognised as risk factors for lower birth weight and prematurity, late sexual debut may at first seem a rather unusual risk. However, the shorter interval between sexual debut and actual pregnancy may increase the risk for active genital infection and hence explain the elevated prematurity rates.

In developing countries tracing and treating gonorrhoea during pregnancy is limited by the frequent absence of symptoms and the difficult implementation of treatment to all positive women. Routine endocervical cultures and treatment for gonorrhoea should be promoted in pregnancy, in addition to routine postnatal ocular prophylaxis as a measure to prevent neonatal complications caused by $N$ gonorrhoeae. As the presence of $N$ gonorrhoeae and other pathogens in the cervix is

Table 3 Genital infection versus premature delivery and mean birthweight. Only significant differences are marked in this table

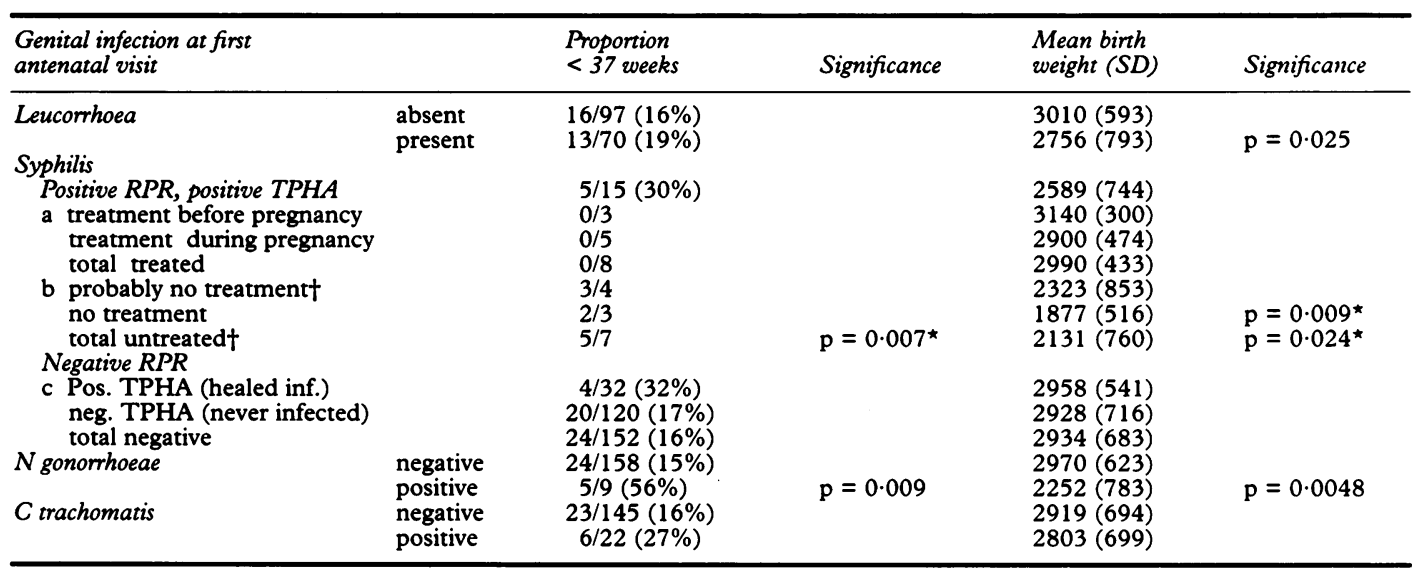

RPR: Reactive Plasma Reagent, TPHA: $T$ pallidum haemagglutination assay. $\dagger=$ received no treatment in our institution, *compared with "total treated".

Table 4 Combined effect on premature birth and birthweight of untreated syphilis and gonorrhoea

\begin{tabular}{|c|c|c|c|c|c|}
\hline Gonorrhoea & Syphilis & Preterm * & $\begin{array}{l}\text { Birth-weight } \\
\text { (SD) }\end{array}$ & $\begin{array}{l}\text { Statistical difference (birth weight) } \\
\text { vs } G-\mid S-\quad \text { vs } G+/ S-\end{array}$ & vs $G-/ S+$ \\
\hline $\begin{array}{l}- \\
\overline{+} \\
+ \\
+\end{array}$ & $\begin{array}{l}- \\
+ \\
+ \\
+\end{array}$ & $\begin{array}{crc}21 / 153 & (14 \%) \\
3 / & 5 & (60 \%) \\
3 / & 7 & (42 \%) \\
2 / & 2 & (100 \%)\end{array}$ & $\begin{array}{l}2955(668) \\
2486(597) \\
2540(647) \\
1245(185)\end{array}$ & $\mathrm{p}<0.00001$ & $p=0.009$ \\
\hline
\end{tabular}

*The effect of combined infection with syphilis and $N$ gonorrhoeae on prematurity rates is greater than can be expected from the sum of the individual contribution of both infections (Mantel-Haenszel confounding): OR $12 \cdot 1$ (CL95 1.7-85.9). When both sum of the individual contribution of both infections (Mantel-Haenszel confounding): OR $\mathrm{R}_{\mathrm{MH}} 12 \cdot 1$ (CL95 $\left.1 \cdot 7-85 \cdot 9\right)$. When
infection occur simultaneously, birth weight is also less than when gonorrhoea $\left(\mathrm{G}+/ \mathrm{S}^{-}\right.$) or syphilis $\mathrm{G}_{-} / \mathrm{S}-$ ) occurs alone. 
usually associated with severely disturbed vaginal flora, ${ }^{417}$ routine Pap smears may be useful to select patients who need further microbiological studies.

Ideally, two specimens from the endocervix or from different sites, such as the urethra, should be taken. By statistical inference one can presume from the duplicate swab series that two out of seven $N$ gonorrhoeae infections would have been missed by taking only one swab. In non-pregnant women a single endocervical swab will recover $90 \%$ of $N$ gonorrhoeae infections, while either two consecutive endocervical swabs or an endocervical plus anal culture would recover over $99 \%$ of infections. ${ }^{13}$ In pregnancy similar data are not available, but some reluctance for adequate sampling of infected material from inside the endocervical canal may account for part of the lower yield of a single swab, as the pregnant condition may predispose for complications like excessive cervical bleeding or traumatic rupture of the foetal membranes. It would be of interest to investigate whether concordant positive swabs correlate to a higher infectious potential of $N$ gonorrhoeae than discordant swabs, thereby possibly leading to more severe pregnancy complications. In the present series no such difference could be proven, but numbers were small.

In univariate analysis, leucorrhoea was significantly associated with low birth weight and an increase in prematurity rates. Recently vaginal discharge caused by bacterial vaginosis was found to be an independent risk factor for preterm labour. ${ }^{5}$ In the present series, however, the association of premature delivery or low birth weight and leucorrhoea was not significant after multi-variate analysis, indicating that the link between leucorrhoea and preterm delivery was probably mediated through gonorrhoea and, to a lesser extent, syphilis.

Inadequate laboratory facilities may hamper the routine practice of endocervical cultures for $N$ gonorrhoeae during pregnancy in many African countries. However, the burden of infection-related prematurity must not be overlooked. Of all premature births among women with gonorrhoea in the present series, $72 \%$ could have been related to $N$ gonorrhoeae. If gonorrhoea were eliminated, prematurity might be reduced by $13 \%$ in this population. Estimating cost of a ThayerMartin culture at $£ 3$, sensitivity of culture during pregnancy at $80 \%$, treatment efficacy at $90 \%$, treatment of uncomplicated $N$ gonorrhoeae infection at $£ 1.5$ per patient, treatment efficacy at $90 \%$, and the mean cost of intensive care at $£ 715$ per premature newborn, the cost-benefit ratio would be 2.9 in favour of routine screening versus no screening in this population on the basis of such direct costs only.

It is concluded that untreated gonorrhoea and syphilis during pregnancy are associated with prematurity and low birth weight. Therefore, in addition to routine RPR testing and ocular prophylaxis of the newborn, swabs from the endocervical canal, in duplicate if possible, should ideally be plated on a Thayer-Martin plate at least once during pregnancy in high risk communities. Although the cost-benefit of this approach seems clearly favourable, practical problems due to inadequate diagnostic facilities warrant a continued search for simple and cheap tracing tools for gonorrhoea and other genital infections during pregnancy in Africa.

We thank Dries Van Straeten (Pretoria, South-Africa) for the practical help, P Hooft (Leuven, Belgium) for the statistical advice, P Piot (Antwerp, Belgium) and J Paavonen (Helsinki, Finland) for the critical review of the manuscript.

1 Gravett MG, Nelson P, DeRouen T, Critchlow C, Eschenbach DA, Holmes $\mathrm{KK}$. Independent associations of bacterial vaginosis and Chlamydia trachomatis infection with adverse pregnancy outcome. $\mathscr{F} A M A$ 1986; 256:1899-903.

2 Kundsen RB, Driscoll SG, Monson RR, Yeh C, Biano SA. Association of Ureaplasma urealyticum in the placenta with perinatal morbidity and mortality. $N$ Engl f Med 1984;310:941

3 Hillier SL, Martius J, Krohn M et al. A case-control study of chorioamniotic infection and histologic chorioamnionitis in prematurity. $N$ Engl $F$ Med 1988;319:972-8.

4 Donders G, Moerman P, De Wet GH, Hooft P, Goubau P. The association between chlamydia cervicitis, chorioamnionitis and neonatal complications. Arch Gynecol Obstet 1991;249:79-85.

5 McGregor JA, French JI, Richter $\mathrm{R}$ et al. Antenatal microbiological and maternal risk factors associated with prematurity. Am $\mathcal{f}$ Obstet Gynecol, 1990, 163: with premat

6 Martius J, Krohn MA, Hillier SL et al. Relationships of vaginal Lactobacillus species, cervical Chlamydia trachomatis, and bacterial vaginosis in preterm birth. Obstet Gynecol 1988;71:89-95.

7 Smith LG Jr, Summers PR, Miles RW, Biswas MK, Pernoll ML. Gonococcal chorioamnionitis associated with sepsis: a case report. Am f Obstet Gynecol 1989;160:573-4.

8 Lacey CJ, Milne JD. Preterm labour in association with Neisseria gonorrhoeae: case reports. Br $\mathcal{f}$ Venereal Dis 1984;60:123-4.

9 Edwards LE, Barrada MI, Hamann AA, Hakanson EY. Gonorrhoea in pregnancy. Am $\mathcal{f}$ Obstet Gynecol 1978;132:637-41

10 Handsfield HH, Hodson A, Holmes KK. Neonatal gonococcal infection. I. Orogastric contamination with Neisseria gonorrhoeae. FAMA 1973;225:697-701.

11 Elliot B, Brunham RC, Laga M, Piot P, Ndinya-Achola JO, Maitha G, Cheang M, Plummer FA. Maternal gonococcal infection as a preventable risk factor for low birth weight. F Infect Dis 1990;161:513-6.

12 Amstey MS, Steadman KT. Asymptomatic gonorrhoea and pregnancy. $\mathcal{F}$ Am Vener Dis Assoc 1976;3:14-6.

13 Sweet RL, Gibbs RS. Infectious Diseases of the Genital Tract, 2nd ed, Baltimore, Williams \& Wilkins, 1990.

14 O'Farrell N, Hoosen AA, Kharsany AB, van den Ende J. Sexually transmitted disease pathogens in pregnant women in a rural South African community. Genitourin Med 1989;65:276-80.

15 Shultz KF, Cates W Jr, O'Mara PR. Pregnancy loss, infant death and suffering: legacy of syphilis and gonorrhoea in Africa. Genitourin Med 1987;63:320-5.

16 Laga M, Plummer FA, Piot P et al. Prophylaxis of gonococcal and chlamydial ophthalmia neonatorum. A comparison of silver nitrate and tetracycline. $N$ Engl $\mathcal{F}$ Med 1988;318:653-7.

17 Donders G, De Wet GH, Hooft P, Desmuyter J. Lactobacilli in Pap smears, genital infections and pregnancy. Am $\mathcal{f}$ Perinatol (in press.).

18 Schoendorf KC, Hogue CJR, Joel MPH et al. Mortality among infants of black as compared with white collegeeducated parents. N Engl F Med, 1992;326:1522-6.

19 Thorp JM, Katz VL, Fowler LJ, Kurtzman JT, Bowes WA. Foetal death from chlamydial infection across amniotic membranes. Am f Obstet Gynecol 1989;161: 1245-6. 